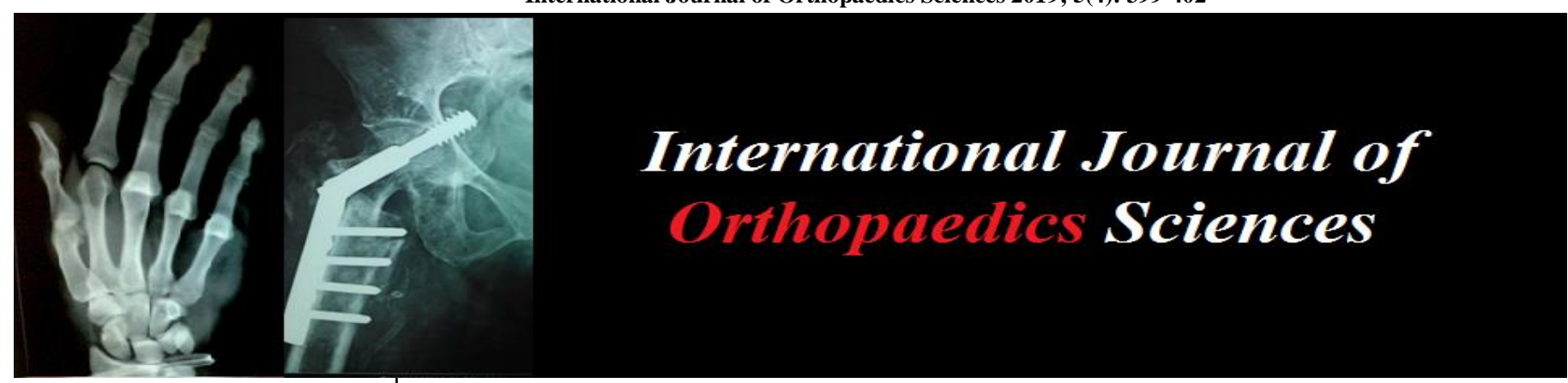

E-ISSN: 2395-1958

P-ISSN: 2706-6630

IJOS 2019; 5(4): 399-402

(C) 2019 IJOS

www.orthopaper.com

Received: 22-08-2019

Accepted: 26-09-2019

\section{Dr. Shivanna}

Assistant Professor, Department of orthopaedics, Chamarajanagar Institute of Medical Sciences Chamarajanagar, Karnataka, India

\section{Dr. Mahadevprasad}

Senior Resident Orthopaedic

Surgeon Department of orthopaedics, Chamarajanaga Institute of Medical Sciences Chamarajanagar, Karnataka, India
Corresponding Author: Dr. Shivanna

Assistant Professor, Department of orthopaedics, Chamarajanagar Institute of Medical Sciences Chamarajanagar, Karnataka, India

\section{Functional outcome of surgical management of intercondylar fracture of distal humerus in adults by olcranon approach- $\mathrm{A}$ retrospective study}

\section{Dr. Shivanna and Dr. Mahadevprasad}

DOI: https://doi.org/10.22271/ortho.2019.v5.i4g.1705

\section{Abstract}

Background: An intercondylar injury after distal humerus are uncommon injuries and present the most difficult challenge of fracture of lower end humerus. There is an increase in the incidence of injury of intercondylar fractures in the younger age population due to fall, road traffic accidents and also in the older population due to fall, increased weight and increased life expectancy and poor bone mineral density. The aim of our study is to analyse the functional outcome of distal humerus intercondylar fractures fixation through olecranon osteotomy and comparing the outcome results with the previous literatures.

Material and Methods: The retrospective study was carried out at our institute, collecting the records retrospectively from 2019 going back upto 2015. Thirty three patients were treated with open reduction and internal fixation with olecranon osteotomy approach. All distal humerus intercondylar fractures were fixed with Locking compression plate and olecranon osteotomy was fixed with tension band wiring, either with $\mathrm{k}$ wire or cancellous screw fixation. These patients were retrospectively assessed using patient documentation like $\mathrm{X}$ rays and charts and clinical examination records. The final outcome then assessed with Rise Borough Radin scoring system.

Results: Fifteen patients belong to age group 21-35yrs followed by 9 were in the age group 50 to 65 yrs, 5 were in the age group 36-50yrs and 4 were more than 65 years. Minimum age was $22 \mathrm{yrs}$ and maximum age was $70 y r s$. Mean age of the study was $43.03+15.71$ yrs. Majority of the subjects $54.5 \%$ were male and $45.5 \%$ were female. Majority of the patients sustained injury due to fall and road traffic accidents. Majority of the patients $(60.6 \%)$ had type 3 fracture followed by $27.3 \%$ had type 2 fracture and $12.1 \%$ had type 4 fracture. Twenty six patients $(78.8 \%)$ did not had any complications, 2 patients had implant breakage, 2 patients suffered superficial infection, 2 patients had ulnar neuropathy and one of the patient had non-union. Most of the patients (48.5\%) had good results followed by $36.4 \%$ had fair results and $15.2 \%$ had poor results.

Conclusion: The present study was conducted to assess the functional outcome of surgical management of Thirty three cases of intercondylar fracture of distal humerus by olecranon approach. Well exposure of distal articular surface of humerus is mandatory to achieve the anatomical reduction of the Comminuted distal humeral fractures. Olecranon osteotomy approach yields better such exposure.

Keywords: Functional outcome, humerus, olecranon osteotomy APPROACH

\section{Introduction}

An intercondylar fracture of the distal humerus are uncommon injuries and present the most difficult challenge to the orthopaedic surgeon in their practice. The complex shape of the elbow joint, the adjacent neurovascular architecture and the sparse soft tissue envelope combine to make these fractures difficult to treat. Acceptable results have been reported in a majority of patients treated by open reduction and Internal fixation ${ }^{[1]}$.

The only reliable method for restoring the normal alignment and contour of the distal humerus is operative exposure and direct manipulation of fracture fragments. However, fixation of fracture fragments must be stable enough to allow motion while ensuring union. In the early and middle parts of twentieth century, operative treatment was combined with de vascularizing exposure, inadequate fixation, and cast immobilization. The result was often elbow stiffness and delayed healing. 
In this context, non operative treatments such as the so-called bag- of -bones technique (a short duration of immobilization in either a cast or a collar and cuff followed by mobilization as tolerated) were established as treatment alternatives ${ }^{[2]}$.

Restoration of painless and satisfactory elbow function after a fracture of the distal humerus requires anatomic reconstruction of the articular surface, restitution of the overall geometry of the distal humerus and stable fixation of the fractured fragments to allow early and full rehabilitation ${ }^{3}$. Restoration of the articular surface of the distal humerus must be nearly perfect and sufficiently rigid to permit early mobilization of the elbow if the results is to be satisfactory. Depending upon the nature of comminution and displacement, open reduction and internal fixation with $1 / 3$ tubular plate, reconstruction plate, Kirschner wire and double tension band wiring, distal humerus locking plate fixation can be done individually or in combination.

The result of operative fixation of fractures of the distal humerus remained unpredictable until improved techniques for the fixation of small, articular fractures as developed by the Arbeitsgemeinschaft fur osteosynthese fragen / association for the study of internal fixation (AO/ASIF) and others were applied. On the basis of the results reported in the more recent series, fixation with two plates at 90 degrees angle with one another has become the standard against which all other treatments are measured. Despite the confidence in operative fixation that believes this shift in treatment preference, these remain challenging fractures to treat effectively and best managed by surgeons with interest and experience in skeletal trauma involving upper extremity. Even the most experienced surgeons, however may be intimidated with certain fracture characteristics, including poor bone quality, fractures involving the distal most aspects of the bone columns, and fragmentation of articular surface in sagittal and coronal planes. Some have even suggested total elbow arthroplasty as an alternative to operative fixation.

Although it is wise to be prepared to perform a total elbow arthroplasty in the event that a complex fracture is not amenable to internal fixation, one must keep in mind the functional limitations and eventual failure associated with total elbow arthroplasty. A surgeon treating a healthy active patient with a fracture of distal humerus should make every attempt to reconstruct and preserve the distal humerus ${ }^{[4]}$.

The quality of elbow function following intercondylar fractures is related to the degree to which normal anatomic relationships are restored. Residual elbow stiffness still remains the worst complication of intercondylar fractures as it is poorly tolerated because of lack of compensatory motions in adjacent joints.

Olecranon osteotomy is used in conjuction with a posterior approach for fixation of condylar fracture, as well as the exposure of distal humerus nonunion. Osteotomy can be either nonarticular or Trans articular non articular osteotomy is used for non articular and transarticular osteotomy is reserved for articular condylar fracture. Advantage of olecranon osteotomy is better exposure of the intercondylar region which aids in better reduction and fixation of fracture. Disadvantage is the nonunion of olecranon osteotomy make up to $5 \%$ of cases hardware is often painful and may require hardware removal. Osteotomy fixation can be done with 6.5 $\mathrm{mm}$ cancellous screw in combination with figure of eight and secure the osteotomy with k- wire and tension band wiring. The aim of the present study is to evaluate the functional outcome of surgical management of intercondylar fracture of distal humerus in adults by olecranon osteotomy approach.

\section{Aim and Objectives}

\section{Aim}

The aim of our study is to examine the anatomical and functional consequences of this technique of exposure on the distal humerus intercondylar fracture and olecranon articular reduction and its union.

\section{Objectives}

1. To determine the type of injury and incidence of different types of injury according to Rise Borough and Raddin classification and its outcome in different age group and sex distribution.

2. Evaluation and assessment of age and sex distribution of distal humerus intercondylar fracture.

3. To assess the immediate and late postoperative complication of osteotomy.

4. To determine the late functional out come

\section{Methodology}

The present study includes 33 cases of intercondylar fracture of distal humerus admitted in our institute between 2019 to 2015.

\section{Inclusion and exclusion criteria Inclusion criteria}

- Patients with closed intercondylar fracture of the distal end of the humerus.

- Patients above the age of $18 y e a r s$.

- Having the sufficient hospital record.

- Entire definitive treatment done in our hospital.

\section{Exclusion criteria}

- Compound fractures of the distal humerus.

- Old malunited fractures of the distal humerus.

- Patients with extra articular distal humeral fracture.

- Patients with distal neurovascular injury.

Total 33 patients were included in study were operated between august 2019 to January 2015 at chamarajanagar institute of medical scinces and teaching hospital, chamarajanagar. Patients hospital records were analysed including chart and $\mathrm{x}$ rays and performa was filled. The functional assessment of the patient was done according to Riseborough and Raddin grading system ${ }^{[5]}$.

Good - A range of motion from a flexion contracture of $30^{\circ}$ or less to atleast $115^{\circ}$ of flexion with or without minor subjective symptoms.

Fair -- Range of motion from flexion contracture of between $30-60$ degrees or atleast 115 degrees of further flexion with or without minor subjective symptoms.

Poor-- A range of motion from a flexion contracture of 60 degrees or more to less than 115 degrees of flexion with or without major subjective symptoms.

Minor subjective symptoms -- mild pain on heavy lifting and aching in damp weather.

Major subjective symptoms -- sufficient enough to limit patients function significantly and make him unable to return to his previous activities or employment.

\section{Discussion}

In our study out of 33 cases out of which 30 cases of intercondylar fractures of distal humerus were treated with distal humerus locking compression plate and 3 cases were treated with reconstruction Plate. Our experience with these methods of fixation has given favourable results. The 
findings, the end result and various other data have been analysed and compared in the following discussion.

In our study fractures were commoner in the second and third decade with average age being 43.03+/-15.71 years (22-70). Our findings are comparable to the study made by Jesse B. Jupiter ${ }^{[6,7]}$. In the study by Jesse B. Jupiter et al. found 57 years as the average age in their series but M. Bradford Henley et al. found 32 years as the average age in their series. In 1994 Kun-chuang Want et al., found 47 years as the average age in their series.

Our series had a male predominance with $54.5 \%$ and $45.5 \%$ female patient which were comparable to Kun-Chuang Wang et al., (1994) study. In our series $51.5 \%$ of the cases were due to direct fall and $45.5 \%$ of cases had road traffic accident and $3 \%$ of the case was due to fall of iron rod. Gabel et al. accounted $100 \%$ of his cases to direct fall. M. Bradford Henley ${ }^{[8]}$ accounted $61 \%$ of his cases to road traffic accident, $39 \%$ due to direct fall. The results of the M. Bradford Henley et al., are comparable with our series.

We accounted about $45.5 \%$ incidence of fractures in right side and $54.5 \%$ of the fracture in left side, which is also comparable to other studies. Left sided predominance is probable due to direct fall injury, left sided predominance which is common in our series. In our series we accounted no cases of fractures of RR type I, 27.3\% fractures of RR type II, $60.6 \%$ fractures of RR type III and $12.1 \%$ fractures of RR type IV. M. Bradford et al. ${ }^{[8]}$ in his series noted about $9 \%$ of fractures of RR type I, $12 \%$ fractures of RR type II, $43 \%$ fractures of RR type III and $36 \%$ fractures of RR type IV. In the RR and our series there were no type-I fractures. In RR series, there were 5 type II cases of which 3 had good, 1 fair and 1 poor results. In our series 9 type II cases were found out of which 5 good and 4 fair results had. There were 12 cases of type III fractures in RR series and 20 in ours. RR series had 4 good, 5 fair and 3 poor while our series had 9 good, 8 fair and 3 poor results. RR series had 11 type -IV fractures of which 3 good, 4 fair and 4 poor results were obtained. Our series had 4 type IV fractures of which 2 good and 2 poor results were obtained.
In our series we had two cases of superficial infection which resolved with appropriate antibiotics. Two cases of ulnar neuropathy was seen which resolved spontaneously after conservative treatment. One patient had a non union, in which Reconstruction plate was removed and internal fixation with two $3.5 \mathrm{~mm}$ Distal humerus locking plate with bone grafting was done. Reconstruction plate breakage occurred in two patients where re-surgery was done, broken reconstruction plate was removed and $3.5 \mathrm{~mm}$ distal humerus locking plates were applied with bone grafting. Henly et al. ${ }^{[8]}$ reported $4 \%$ superficial infection $7 \%$ of ulnar neuropathy, $5 \%$ of implant failure, $2 \%$ of non union and $4 \%$ incidence of heterotrophic ossification. Pain in the elbow, shoulder and elbow stiffness are treated with Physiotherapy. Pin neuropraxia and ulnar neuropathy resolved conservatively. Henly et al. ${ }^{[8]}$ reported $4 \%$ superficial infection $7 \%$ of ulnar neuropathy, $5 \%$ of implant failure.

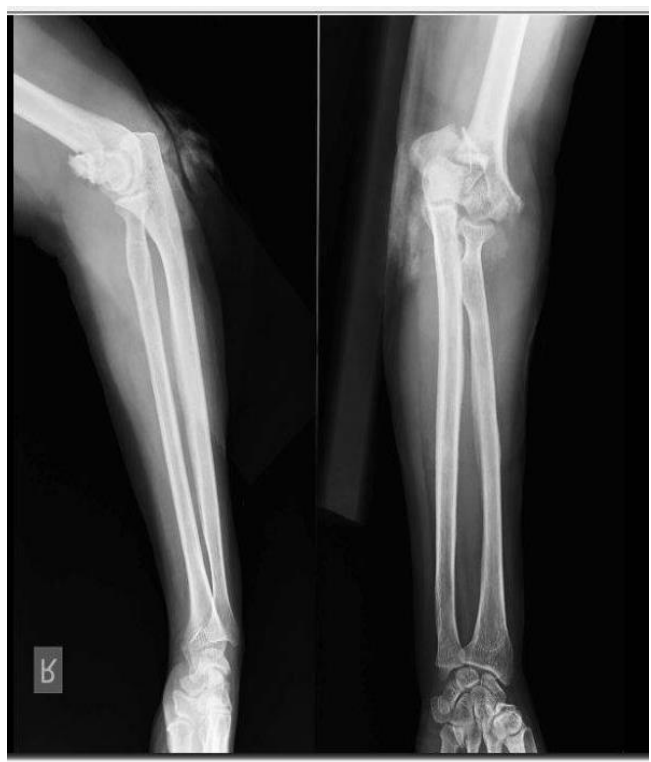

Preoperative $\mathrm{x}$ ray

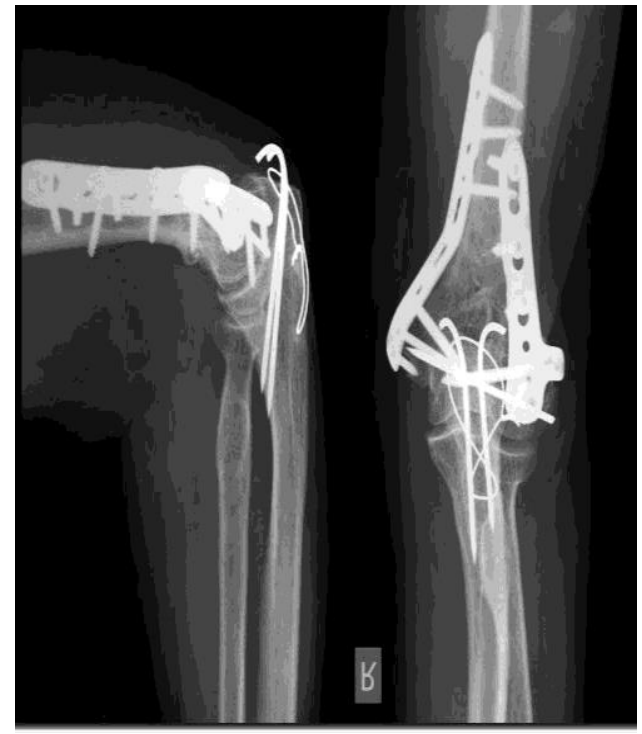

Post of X Ray, Olecranon Osteotomy Fixed with Tbw With K Wire

\section{Conclusion}

The present study was conducted to assess the functional outcome of surgical management of Thirty three cases of intercondylar fracture of distal humerus by olecranon approach.
We conclude

- Inter condylar fractures of the distal humerus are commoner in Second and third decade of life with male predominant in high incidence of fracture due to outdoor activity. 
- The mode of injury was direct fall on elbow or road traffic accidents.

- Inter condylar fractures demands careful evaluation, classification of fracture type and pre-operative planning.

- Trans-olecranon approach provides best visualization of articular surface. There were no complications of olecranon osteotomy.

- Minor complication associated with olecranon osteotomy will be present but for good anatomical reduction and functional outcome this approach is useful.

- Open reduction internal fixation should be done as early as possible. Delay in open reduction internal fixation with delayed soft tissue dissection leads to increased chances of elbow stiffness due to periarticular fibrosis.

- Operative treatment with rigid anatomical internal fixation should be the line of treatment for all grades of Riseborough Radin intercondylar fractures, more so in young adults as it gives best chance to achieve good elbow function.

\section{Summary}

The study included 33 patients from the age of 22 to 70 years of age, the mean age was $43.03+$ or -15.71 yrs. Maximum number of patients were between second and third decade. Sex distribution and side of the limb involvement was not much significant. Predominantly the cuase of the facture is either fall or road traffic accidents. Majority of the patients $60.6 \%$ had type 3 fracture followed by $27.3 \%$ had type 2 fracture and $12.1 \%$ had type 4 fracture. Complications were encountered in very few patients. There was no olecranon non union. Good results found in $48.5 \%$ followed by $36.4 \%$ had Fair result and $15.2 \%$ had poor result. No Statistically Significant correlation found between age, sex, side, type of fracture as compared to previous literatures.

\section{References}

1. Kuntz Jr, David G, Baratz ME. Fractures of the elbow. Orthop Clin North Am Jun. 1999; 30(1):37-61.

2. Jupiter JB, Neff U, Holzach P. Intercondylar fractures of the humerus: An operative approach. J Bone Joint Surg Am. 1985 67:226-238.

3. O'Driscoll SW, Sanchez SJ, Torchia ME. Management of the smashed distal humerus. Orthop Clin North Am Jan 2002; 33(1):19-33.

4. Holdsworth BJ, Mossad MM. Fractures of the adult distal humerus: Elbow function after internal fixation. J Bone Joint Surg Br. 1990; 72B:362-368.

5. Riseborough EJ, Radin EL. Intercondylar $\mathrm{T}$ fractures of the humerus in the adult. A comparison of operative and non-operative treatment in twenty nine cases. J Bone Joint Surg. 1969; 51A:130-141.

6. Jesse BJ. The surgical management of intraarticular fractures of the distal humerus. Chapter-4 in Master techniques in orthopaedics surgery, 1994. p.53-70.

7. Jesse BJ, Urs Neff, Peter H. Intercondylar fractures of the humerus. J of Bone \& Joint Surg. 1995; 67(2):226-239.

8. Henley BM. Intra-articular distal humeral fractures in adults. Orthop Clin North Am. 1987; 18(1):11-23 